\title{
Nasogastric Tube: Spectrum of errors and ways to minimize them
}

\author{
K.C. S
}

Shreeyanta K.C. ,MBBS, Kathmandu Medical College and Teaching Hospital, Sinamangal, Kathmandu, Nepal

\begin{abstract}
Tasogastric tube has been a frequently used 1 method of alleviating gastrointestinal symptoms ${ }^{1}$.Yet placing nasogastric tube is not without complications especially due to displacement of tube or due to placement of tube in wrong tract. During my clinical rotations after passing final MBBS, I frequently encountered difficulty in correct positioning despite trying ideal steps, might be due to uncooperative patients especially in neurologically ill and in children. If one doesn't know the correct steps in beginning of their rotations, then definitely it is troublesome. With time, I eventually discovered that experience matters as well.
\end{abstract}

When I felt we would not get a scheduled lecture or skilled classes for minor procedures, I ended up searching correct procedure guidelines in the internet and it was not surprising to find so many articles about the errors while placing nasogastric tubes and its complications. Recent study suggests that more than 50 percent of the nasogastric tubes could inadvertently migrate from stomach or removed by the patient even after they are confirmed in position by chest $\mathrm{x}$-ray, suggesting that nasogastric tube placement is a procedure to be performed with utmost care ${ }^{2}$. Another study reveals that between September 2011 and March 2016, 95 incidents were reported to National Reporting and Learning System of patient death of Doncaster and Bassetlaw Teaching Hospital NHS Foundation Trust in England, where fluid and medication were introduced to respiratory tract or pleura via misplaced nasogastric or orogastric tube ${ }^{3}$.

The issue attracted the internet in May 2013, when a 52 year old patient was intubated with an NG tube following a car accident. Medical person noticed there was blood in the tube and they treated the patient for

Address for correspondence

Dr. Shreeyanta K.C.

MBBS

Kathmandu Medical College and Teaching Hospital

Sinamangal, Kathmandu, Nepal

E-mail : shreeyantakc@gmail.com gastrointestinal bleeding. It was only after when skull $x$-rays was ordered and doctors realized that the tube was in fact in patient's brain, thankfully the patient recovered following surgery ${ }^{4}$. The common misplacement is into the trachea, which is life threatening if not recognized. Similarly, tube with feeding ports in esophagus increases the risk of aspiration, while in neonates even gastric perforation can occur when the tube is placed blindly ${ }^{5,6}$. Misplacement can occur even with experts, it is more important to invariably check the correct placement and obviously an x-ray, whenever in doubt.

Besides faults in misplacement, I felt that inappropriate feeding and medication errors while giving them via NG tube are also of concerns that one must take care of. NG tube is also common method of delivering oral medications when patients cannot swallow safely. Incorrect administration may result in clogged feeding tube, decreased drug efficacy or drug-nutrient interactions. In prolonged NG tube use, especially in neonates, nasal ala pressure sore has been reported. It is a minor and easily ignored, but is avoidable ${ }^{7}$.

To minimize all these errors, first and foremost one must first know the correct indications of an NG tube. Patients who are unable to eat and do not have absolute contraindications are candidates for enteral tube feeding. Besides, they are also used for aspiration of gastric contents and for decompression in abdominal distension. The preference of enteral over parenteral nutrition is based on numerous studies showing that enteral nutrition is associated with fewer infection, especially pneumonias ${ }^{8}$. Absolute contraindications include complete bowel obstruction, bowel ischemia, ileus and ongoing circulatory shock with high vasopressor requirement, maxillofacial trauma and surgeries, skull fractures, oesophageal tumors fistula or surgeries, head and neck surgery and patient with known coagulopathy or is receiving anticoagulant medication or is known to have esophageal varices without first taking advice from senior medical staffs 8 . For correct placement of an NG tube, I recommend every 
beginners like me to go through procedural guidelines and some really catchy videos on web rather than blindly practicing yourself.

After performing the procedure a number of times under senior's supervision, one would definitely be more confident, but I was concerned what to do if there any complications arose. Luckily misplacement is the only fault that I have made till today but then I looked for other possible issues and found them to be relevant to share, like risks of aspiration, faulty feeding leading to diarrhea, not meeting nutritional requirement, errors in medication and nasal ala pressure sores in prolonged NG placement. In addition, NG tube, once placed should also be checked every time while assessing a patient for blockage; adhesive area should be checked for any pressure trauma and if kept for decompression then make sure it is not blocked. It should be flushed or a new tube should be used if not functioning properly.

To reduce risk of aspiration via NG tube, all intubated patients receiving enteral nutrition, the head of bed should be elevated 30-45 degrees, prokinetic agents like metoclopramide should be initiated when feasible? Diarrhea in enterally-fed patients should be promptly

\section{REFERENCES}

1. Makama JG. Uses and hazards of nasogastric tube in gastrointestinal diseases: An update for clinicians. Ann Nigerian Med[Internet]. 2010;4:37-44[Assessed on 25 June 2018]. [FullText]

2. Borsci S, Buckle P, Huddy J, Alaestante Z, Ni Z, Hanna $\mathrm{GB}$. Usability study of $\mathrm{pH}$ strips for nasogastric tube placement. PLoSONE[Internet]. 2017November30 [Cited 20 June 2018];12(11):e0189013. [DOI]

3. Doncaster and Bassetlaw Teaching Hospitals, NHS Foundation Trust. Nasogastric tube management and care. United States: Doncaster and Bassetlaw Teaching Hospitals, NHS Foundation Trust [Internet]; 2017[Cited 1 July 2018]. [FullText]

4. Hendricks D. Recent Deadly NG Tube Error Causes Internet Sensation. HealthTechZone[Internet]. 2013 May 20 [Cited 21 June 2018].[Full Text]

5. Metheny NA, Meert KL, Clouse RE. Complications related to feeding tube placement. Current opinion in gastroenterology. 2007March;23(2):178-82. [DOI] investigated for hyperosmolar medications like sorbitol, electrolyte losses and other infectious causes. One should make sure that patient meets his caloric requirement by calculating the required values using standard formulas that are available in texts.

When medicines are delivered via NG tube, one must consider appropriate tube size and appropriate drug doses forms. Before administering it must be determined whether the medicines are suitable for crushing or opening the capsule. Feeding tube should be properly flushed before and after each medication is administered. To minimize drug-nutrient interactions, special considerations should be taken while administering phenytoin, carbamazepine, warfarin, fluoroquinolones and proton pump inhibitors via feeding tube ${ }^{10}$.

\section{CONCLUSION}

NG tube appropriateness needs cooperation between physicians, nurses and patients, acknowledging precautions starting from insertion to administering feed and medications. Case reports on enteral feeding errors should be routinely sought and acknowledged to prevent the same in their practices.Though with time anyone would be confident, but why not enlighten the risks and ways to get through it at the earliest?

6. Mollitt DL, Schullinger JN, Santulli TV. Selective management of iatrogenic esophageal perforation in newborn. Journal of plastic surgery. 1981December;16(6):989-93. [PubMed]

7. Hamidi M, Sheikhzadeh D, Sharabiani BA. Nasogastric Tube placement errors and complication in paediatric ICU: A case report. Journal of Cardiovascular and Thoracic research. 2011;3(4):133-4.[DOI]

8. Marino PL. The ICU Book. $4^{\text {th }}$ Edition. Philadelphia: Wolters Kluwer, Lippincott William and Wilkins;2014. [FullText]

9. Martindale RG, McClave SA, Vanek VW, McCarthy M, Roberts P, Taylor B,Ochoa JB, Napolitano L, Cresci G. Guidelines for the provision and assessment of nutrition support therapy in the adult critically ill patient: Society of Critical Care Medicine and American Society for Parenteral and Enteral Nutrition. J Parenter Enter Nutr. 2009MayJune;33(3):277-316.[DOI]

10. Williams NT. Medication administration through enteral feeding tubes. Am J Health Syst Pharm. 2008Dec 15;65(24):2347-57.[DOI] 